\title{
LEVERAGING SPATIAL MODEL TO IMPROVE INDOOR TRACKING
}

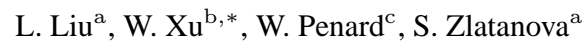 \\ ${ }^{\text {a }}$ Delft University of Technology, Julianalaan 134, 2628BL, Delft, the Netherlands - (1.liu-1, s.zlatanova) \\ @ tudelft.nl \\ ${ }^{\mathrm{b}}$ Fugro Intersite, Dillenburgsingel 69, $2263 \mathrm{HW}$, Leidschendam, the Netherlands \\ W.Xu@fugro.nl \\ ${ }^{\mathrm{c}}$ CGI Nederland, George Hintzenweg 89, Rotterdam, the Netherlands \\ w.penard@cgi.com
}

KEY WORDS: Spatial Model, Indoor Tracking, Semantics, Topology

\begin{abstract}
:
In this paper, we leverage spatial model to process indoor localization results and then improve the track consisting of measured locations. We elaborate different parts of spatial model such as geometry, topology and semantics, and then present how they contribute to the processing of indoor tracks. The initial results of our experiment reveal that spatial model can support us to overcome problems such as tracks intersecting with obstacles and unstable shifts between two location measurements. In the future, we will investigate more exceptions of indoor tracking results and then develop additional spatial methods to reduce errors of indoor tracks.
\end{abstract}

\section{INTRODUCTION}

Nowadays indoor navigation heavily relies on an accurate and stable positioning or localization technique. Unfortunately, existing positioning techniques are still at experimental phase (Fuchs et al., 2011; Miller, 2006). Compared with outdoor GPS tracks (recordings of positions at regular intervals), indoor tracking suffers from low accuracy, which results in a limited number of indoor tracking applications.

Although we may not get highly accurate position information in an indoor environment, tracking pedestrians still appears attractive, especially to some mission-critical scenarios (Fuchs et al., 2011). For some applications users may be more interested in moving trends than accurate coordinates.

Existing indoor positioning and navigation experiments (Miller, 2006; Spassov, 2007) show that under some conditions users (human or robot) can navigate even if individual localization accuracy may not be very high.

Meanwhile, different indoor tracking methods (Burgard et al., 1997; Girard et al., 2011; Jensen et al., 2009; Khider et al., 2012; Miller, 2006; Spassov, 2007; Thrun et al., 2005) design various strategies to avert deviation of tracks. However, they largely rely on positioning equipment, sensors and users. The results reveal varying properties in precision (Fuchs et al., 2011).

Compared with the uncertain results from combining various hardware, a more stable configuration can be achieved if the properties of the spatial model are used. The information stored in spatial models, such as coordinates, semantics of objects, topological relations between objects, etc. can provide some qualitative support for indoor localization regardless the used hardware.

In order to make a better use of indoor positioning measurements and be independent from the adopted devices, this paper is going to present a suitable spatial model and related information to qualitatively improve indoor tracking results.

\section{BACKGROUND}

To be able to localize a person or robot in a given indoor area, the indoor space has to be partitioned. Indoor space can be par- titioned by either real building boundaries (walls) or artificial boundaries, which are the result of a subdivision/decomposition procedure. Such artificial subdivision/decomposition can be based on a regular grid (grid for short), triangulation tessellation, trapezoidalbased tessellation and Voronoi diagrams (Afyouni et al., 2013).

Grid is widely applied to indoor navigation and tracking. Li et al. (Li et al., 2010) elaborate on a grid graph model. They first overlay the building parts/ cellular units (such as a room, a wall, etc.) with grids and then generate a grid graph. The underlying cellular units provide semantic information to the corresponding grid cells. One grid cell of the grid graph has one and only one membership of a cellular unit, and the topological relationships among cellular units can be represented by the edges of the grid graph.

Similarly, in robot motion, a planning occupancy grid approach uses a regular matrix of equally-sized cells for autonomous navigating robots (Franz, 2005; Moravec \& Elfes, 1985). In this matrix, each cell connects to its eight neighboring cells (with exception of boundary cells). A high probability value is assigned to grids in accessible/navigable spaces and a low value to grids occupied by objects.

In addition to subdivision/decomposition approach, semantic and topological information is important as well for indoor navigation. The topology of an indoor space can be modeled in either a 3D space or 2D layers (Worboys, 2011). Two types of topology are distinguished: connectivity and adjacency. Most existing spatial models utilize connectivity graph to represent indoor space topology (Domnguez et al., 2011). The semantics of a spatial model describes the basic spatial and structural concepts of indoor environments (Liu \& Zlatanova, 2012; Tsetsos et al., 2006). Semantics is also referred to ontology when it is utilized for reasoning (Worboys, 2011).

With spatial information, one can conduct indoor tracking with different localization devices. Commonly indoor tracking methods includes: Dead reckoning, Grid filter, Map matching, Modelbased approaches.

Dead reckoning (DR) computes a persons current location by advancing a known position with course, speed, time and distance 
to be travelled. DR data can be collected by inertial measurement unit (IMU) on tracking devices. The uncertainty of dead reckoning positions grows with time thus it is necessary to check the position regularly (Miller, 2006).

Grid filter is a kind of discrete Bayesian filter, which probabilistically estimates a targets location based on observations from sensors. This type of methods are widely used in the field of robotics (Burgard et al., 1997; Thrun et al., 2005). They computes the location in two phases: the prediction phase where the prior probability of location is estimated based on the previous location, a motion model and the map of tracking environment; and the update phase where the posterior probability is computed by multiplying the prior probability with a conditional probability. The conditional probability is computed according to the measurements of sensors.

Map matching assumes a user can be only located along certain routes. Some constraints on indoor environments are applied to refine estimates of the moving positions of a person inside a building. For instance, a user does not pass through walls, but only along corridors and through doorways (Miller, 2006). Basically, there are two map matching techniques: point-to-vertex matching (i.e. a measured location to a vertex in route), and point-to-edge matching (i.e. a measured location to an edge in route). An implementation of point-to-edge matching shows satisfied results in corridor environment (Spassov, 2007).

Model based methods adopt a vector model of the indoor environment to improve the estimation of user location. This method can be taken as an extension of map matching methods. They consider model features (such as walls or obstacles) (Girard et al., 2011), sensor information (e.g. speed \& direction), and information from users (e.g. mean velocity and velocity variance (Khider et al., 2012)). Jensen et al. (Jensen et al., 2009) proposes a base graph model for tracking which represents the connectivity and accessibility of indoor space.

However, the existing tracking methods employ limited spatial information. To the best of our knowledge, indoor tracking research is seldom focus on integration of geometrical, topological and semantic features of indoor environments for tracking.

Based on used techniques, current types of localization systems include Angulation (angle), Lateration (distance), Fingerprinting, Inertial and motion sensors, and Neighborhood (Fuchs et al., 2011). However, we focus on location data processing rather than specific localization technique. Therefore, in this research we assume the location data are acquired and we aim at mitigating tracking errors in the data.

We take WiFi fingerprinting localization system for example: it may result in three types of tracking errors: a measured location at an incorrect space/wall/table, an incorrect moving direction between some localization results, or sudden jumps between locations (shifting back and forth) (Besada et al., 2007).

We intend to bridge spatial model and indoor tracking and show the capability of spatial model on improving indoor tracking. The grid model is chosen because it simplifies the localization. Meanwhile, geometric, topological and semantic features of the grid model are utilized to estimate the probability of a users location.

\section{METHOD}

This section presents how an appropriate spatial model can help on improving indoor tracking results. We concentrate on two major errors, i.e. 1) a track crosses indoor objects and 2) a track jumps back and forth.

\subsection{Using Spatial Model}

As we mentioned, we decided to adopt grid model due to its regularity and flexibility. Meanwhile we take motion direction of pedestrian into consideration as well.

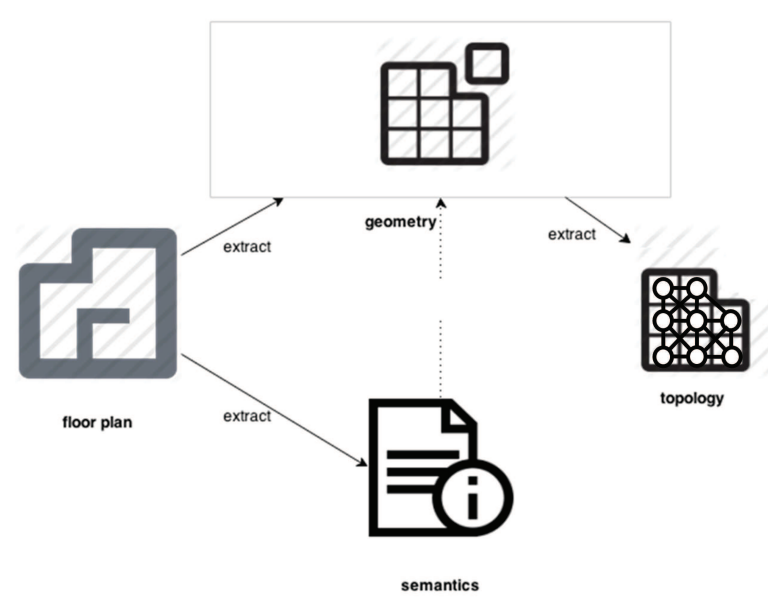

Figure 1: Spatial model construction

In the first place, we require a spatial model incorporating geometry, topology and semantics of indoor environments. In order to detect unrealistic locations, the geometry, topology and semantics of a spatial model need to be used together. Figure 1 provides the workflow of constructing the required spatial model.

Firstly, we pick digital floor plans of a building; secondly, from the original data, we extract semantics of spaces (e.g. room, door); thirdly, we keep the vector geometry of indoor spaces and discretize it to grids; fourthly, we integrate semantics and grids so that each grid cell has a clear meaning or membership; Finally, according to the computed grid model of indoor spaces, we can generate the topological model (i.e. connectivity) of the grid model.

In the next subsections, we will explain each type of information and the usages of them for tracking.

3.1.1 Geometry At the beginning we need to link a measured location to the grid model we made. Thus we tag the center of grid with accurate coordinates. Afterwards, each measured location is mapped to its closest grid (i.e. the point-to-vertex way of map matching).

Besides coordinates, a buffer of a location is applied as well. The relevant part for a location would possibly be the deviation area of the measurement of the location (Fig. 2a). Thus a buffer of the known previous location is used to represent the search region of the current location. The size of the buffer depends on walking speed and the time interval of location determination. This implies that the human must be in the buffer area in this given time interval.

Two types of distances are introduced to estimate the correctness of the new positions: Euclidean distance and Manhattan distance. The well-known Euclidean distance is the direct length between two points in Euclidean space; while the Manhattan distance represent the sum of the absolute differences of their Cartesian coordinates (Wikipedia, 2014b) (Fig. 2b). In addition, we use an orientation vector to represent the direction from one grid cell to another (Fig. 2c).

As mentioned above, the buffer of previous computed location is used to infer the current possible location. Except the buffer, the 


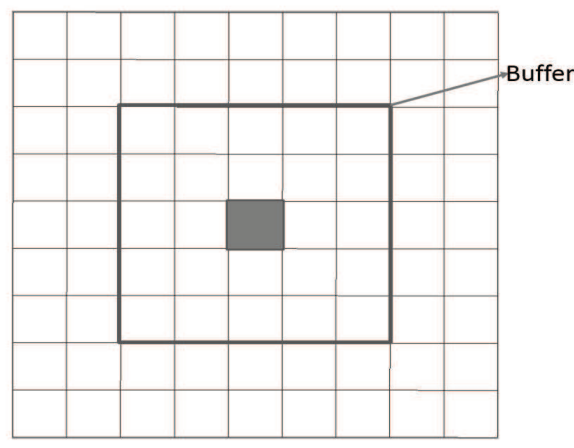

(a)

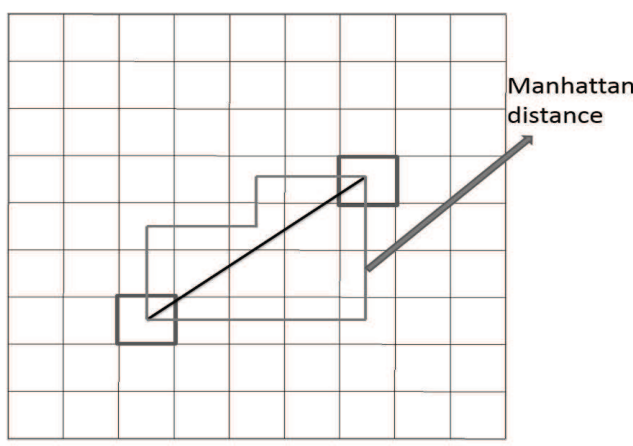

(b)

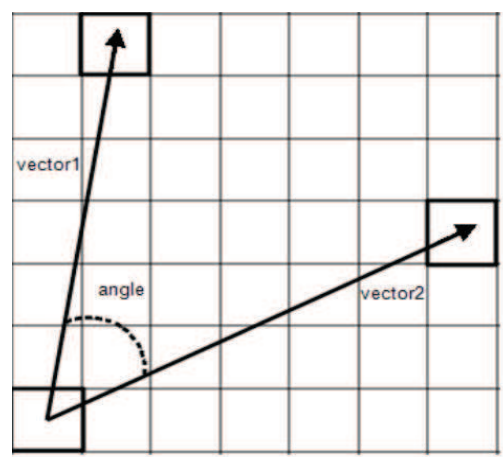

(c)

Figure 2: Buffer, Distance and Direction

previous direction and the difference of the two types of paths (Euclidean and Manhattan) are taken into consideration to compute the new location.

3.1.2 Semantics We pick out important semantics for tracking, namely, floor, space, door, and obstacle. Floor helps us to know the switch of floors; space includes room, corridor/passage and vertical passage.

We define space as a region with real boundary (e.g. walls), or a region with specific function. Space is a quite important notion since user expects to be localized in a correct space even without accurate location inside of the space.

Door is a connection between two separate spaces, namely, it is the transition from one space to another. It might happen that a user may obtain many inaccurate measurements around a door. In this case its pivotal to be aware which room the current location is.
Obstacle is defined for objects or regions, which are occupied and inaccessible. It provides constraints that a users location should not be located inside an obstacle, and the obstacle should not on a users track. In this manner, we obtain tracks outside of obstacles, i.e. avoiding them.

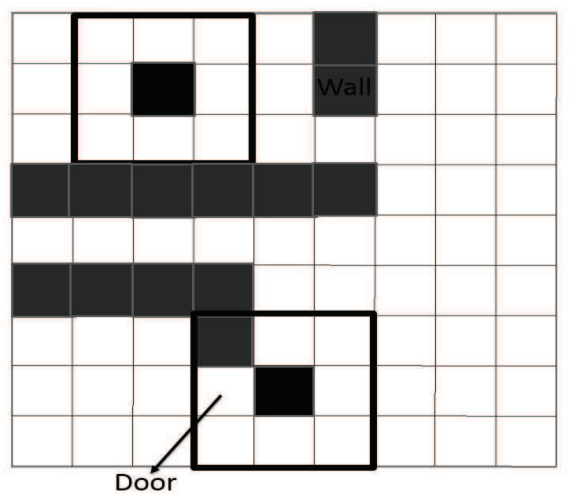

Figure 3: Neighborhood in a space and between spaces

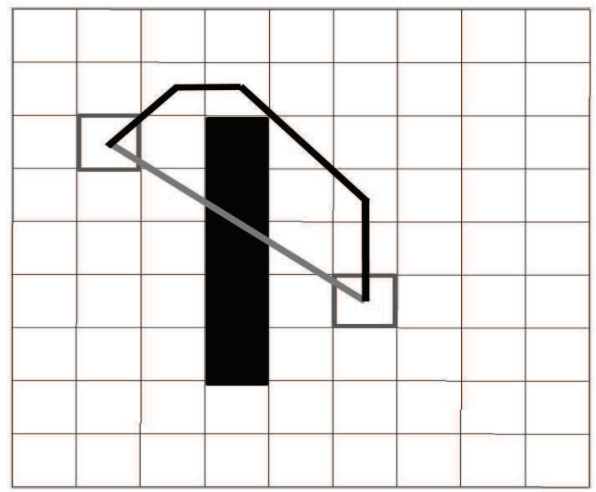

Figure 4: The difference between Manhattan shortest distance and Euclidean shortest distance

3.1.3 Topology As connectivity indicates whether a grid cell can be reached from a predefined location, its an ideal indicator for tracking. If the neighboring grid cells do not belong to any obstacle and are in the same space to a grid, then the grid connect with its neighbors. In this paper we select 8-neighborhood, which means there are at most 8 connected neighbors to one grid cell. If a grid cell and one of its neighbors are not in the same space, then they may connect by grid cells of a door between them (Fig. $3)$.

Based on connectivity between different grid cells, its easy to get the shortest path between two connected grid cells by employing shortest path algorithms. It is worth noting that the shortest path represents Manhattan distance.

Given two grid cells, we compute the difference between their Manhattan shortest distance and Euclidean shortest distance. The difference can indicate obstacle occurrence. If there is no obstacle between two grid cells, the difference of the two distances will be very small. Otherwise, the difference will increase due to obstacle avoidance (Fig. 4).

\subsection{Improving Tracks}

After we introduce the geometric, topological and semantic features of the grid model for indoor tracking, we can make full use of them. For instance, a location is taken as a possible candidate when it is accessible (semantics), connected to previous location 


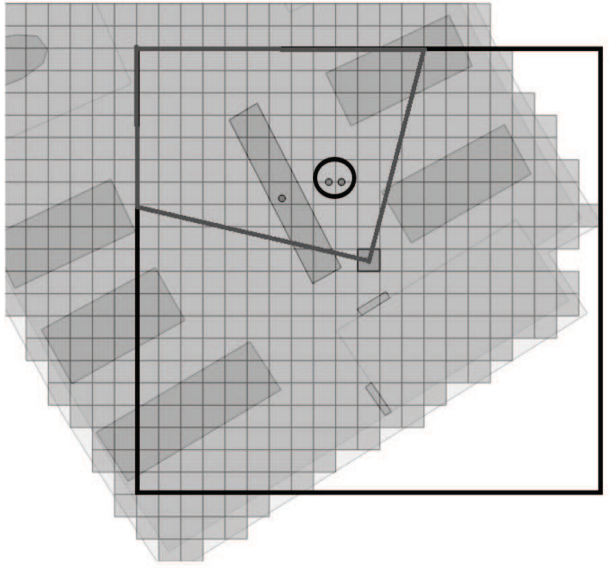

Figure 5: The Selection of measured locations

(topology), and inside a certain buffer of previous determined location (geometry).

In general, by using the previous location and previous moving direction, we could compute the probability of each grid cell to be the current location. Then we can apply the probability to a set of measured candidates of the current location. Finally the one with the highest probability is picked out.

The computation steps are as follows: Firstly, a buffer of the previous location is set up, only the grid cells inside the buffer are considered. Secondly, the probability of these grid cells are assigned. If a grid cell belongs to an obstacle, its probability is set to 0 ; or if the grid cell is at a distinct space from the space of the previous location, its probability is half decreased. Additionally, the difference of the two types of distances is applied. We assume that there is a negative relation between the distance difference and probability, therefore a grid cell with a larger distance difference is assigned a lower probability.

Thirdly, we filter the measured locations. If we dont have previous direction, then a moving direction is computed by averaging orientations of all location measurements at a current moment. In order to determine whether a grid cell is inside the moving direction area, we compare the angle between the orientation vector of the grid cell and the moving direction with an angle threshold. Thus if a measurement is inside the moving direction and not in obstacles, then it is selected (points in circle in Fig. 5). In other words, the candidate set is narrowed down.

In addition, we check the selected candidate locations on their moving directions. The moving directions are computed based on their connection with the previous location. In this manner, improper directions, such as the path crossing an obstacle, can be avoided.

To summarize, we employ a probabilistic method to represent the effect of spatial factors on filtering location measurements, and then pick out the most possible one. Afterwards, the computed or picked candidate locations compose a track which doesnt violate spatial constraints (e.g. no crossing over obstacle).

\section{EXPERIMENT}

\subsection{Preparations}

In this experiment a WiFi positioning system is provided by researchers of Wuhan University (Verbree et al., 2013). The system leverages WiFi fingerprinting method to localize mobile clients. In addition, we employs a magnetometer to measure orientations of a walking person.

The experiment environment is at the office of CGI Company, Rotterdam. The original data to build grid model are floor plans of CGI office. We discrete indoor spaces and objects to grid cells by intersecting the grid cells with floor plans. The grid cell size should be compatible with walking human speed. We chose $0.7 \mathrm{~m}$ because the averaging walking speed of human is $1.4 \mathrm{~m} / \mathrm{s}$ (Wikipedia, 2014a).

We hold two important assumptions during this experiment:

- Start location is known.

- Motion is in a constant pace.

In the next subsections, we will illustrate two cases of tracking. One is about walking inside a space, the other one focuses on passing between different spaces.

\subsection{Case Studies}

The first case is walking inside a large space. We visualized both actual motion track and measured locations of WiFi system in Figure 6a. The problems in the case include locations crossing obstacles/spaces, or even inside of obstacles (Fig. 6a). As shown in Figure 6a, the sequence of measured location are disordered, and there are some measurements located in another space, which contradicts to the real motion track.

Figure $6 \mathrm{~b}$ presents the improvement on track after spatial model aid. The nodes represent the most possible location at each measured moment. The track is significantly improved. Errors such as inside/crossing obstacles/another space, and the wrong moving directions are now corrected.

The second case is walking between spaces. In this case, we would like to know if the correct room is selected especially around doors.

In order to distinguish the room a measurement belongs to, two conditions are proposed for determination of transferring between spaces:

- The door connecting two spaces is in the moving direction area;

- The previous computed location is at the door.

Only when the both conditions are met, the current location is confirmed in the new space (room).

We conducted a test between two spaces. Figure 7a presents the original measured locations and the actual movement route. The locations appear disordered. Figure $7 \mathrm{~b}$ provides the improvement on the original track, and related spaces are highlighted in bold lines. Though the track includes fake zigzags due to measurement errors, the moving trend is still correct and the track doesnt lie in any obstacle. More importantly, the switching between the two spaces was detected. Around the door between the two rooms, we successfully eliminate sharp shifts (back and forth). The locations after processing are strictly successive in a time sequence. 


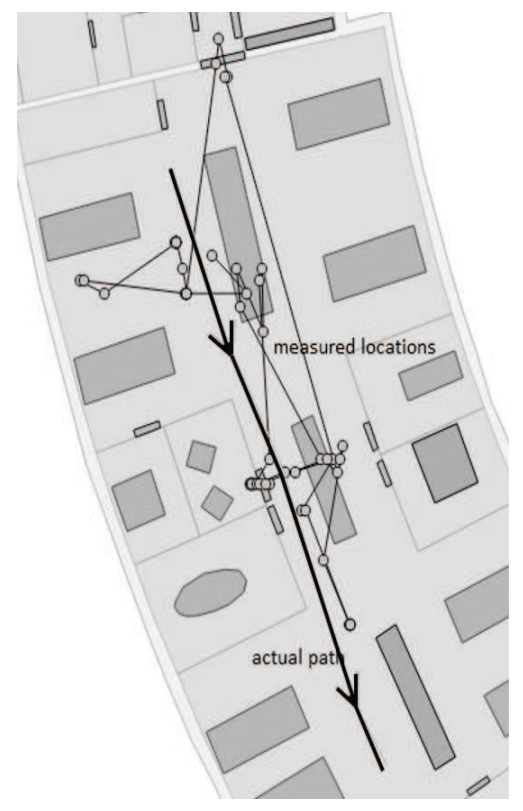

(a) before improvement

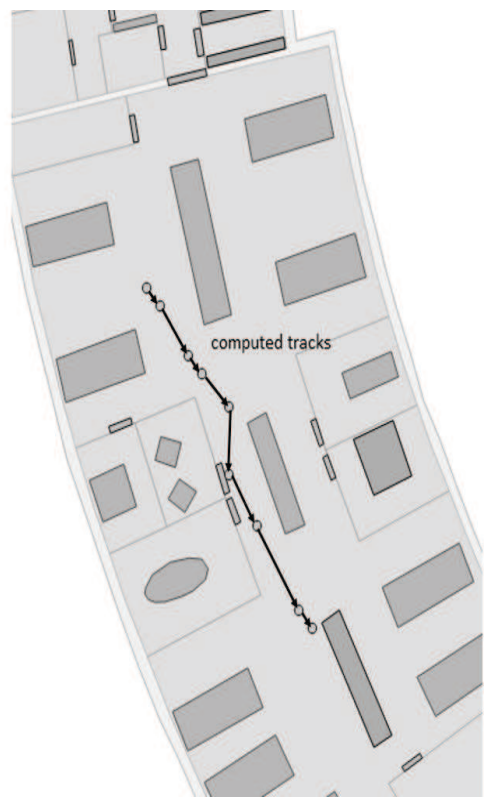

(b) after improvement

Figure 6: Walking inside a Space

\section{CONCLUSIONS}

This paper presents the usage of spatial model and related information for indoor tracking. With WiFi measurements on locations, we leverage a spatial model to qualitatively improve indoor tracking results. Our experiment demonstrates using spatial model has the potential to overcome problems such as tracks over obstacles and unstable shift between two measurements.

In the future, we will investigate more error cases during localization process. Once we collect these exceptions from tracking, we can devise more spatial constraints within grid model. Our final goal is to develop a methodology, which can mitigate tracking errors with only spatial constraints. In this manner, different measurements from distinct localization systems can be processed in a standard method, and thus we can provide improved/smooth tracks for different localization equipment.

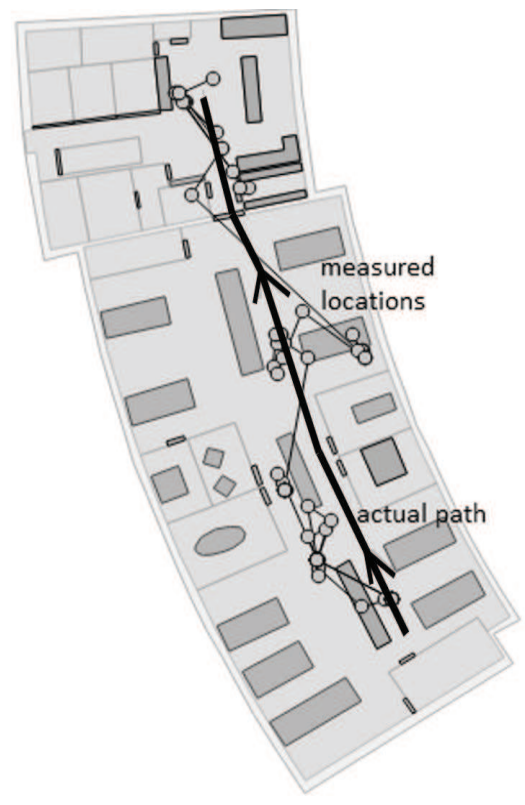

(a) before improvement

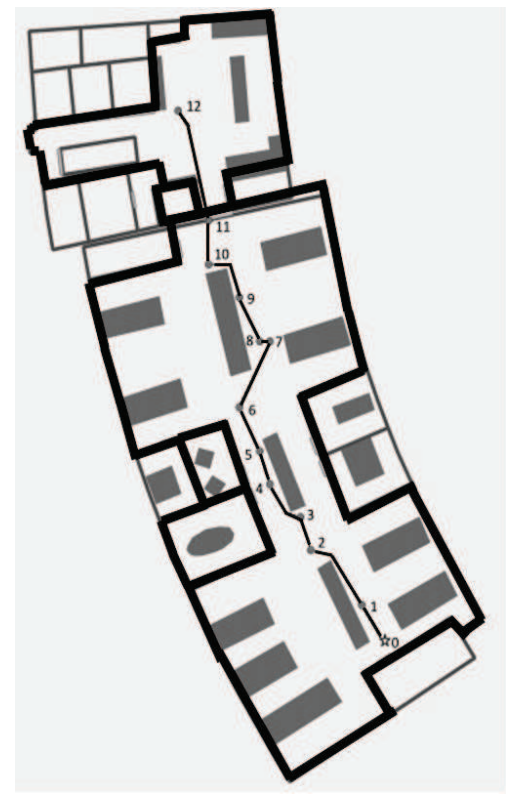

(b) after improvement

Figure 7: Walking between Two Spaces

\section{ACKNOWLEDGMENTS}

Our thanks to Haojun, A., Taizhou, L., Jianjian, W., Menglei , Z. of Wuhan University for allowing us to using the WiFi positioning system they had developed, and thanks to CGI company at Rotterdam for allowing us to setting up test environment in their offices.

\section{REFERENCES}

Afyouni, I., Ray, C., \& Claramunt, C., 2013. Spatial models for context-aware indoor navigation systems: A survey. Journal of Spatial Information Science (4): 85-123.

Besada, J.A., Bernardos, A.M., Tarro, P., \& Casar, J.R., 2007. Analysis of tracking methods for wireless in door localization. In: ISWPC'07 Wireless Pervasive Computing, 2007. 
Burgard, W., Fox, D., \& Hennig, D., 1997. Fast grid-based position tracking for mobile robots. In KI-97: Advances in Artificial Intelligence: 289300 . Springer.

Domnguez, B., Garca, .L., \& Feito, F.R., 2011. Semantic and topological representation of building indoors: An overview. In Proc. of Joint ISPRS workshop on 3D city modelling \& applications and the 6th 3D GeoInfo, Wuhan.

Franz, G., Mallot, H., Wiener, J., \& Neurowissenschaft, K., 2005. Graph-based models of space in architect ture and cognitive science-a comparative analysis. In Proceedings of the 17th International Conference on Systems Research, Informatics and Cybernetics: 3038.

Fuchs, C., Aschenbrucka, N., Martinia, P., \& Wienekeb, M., 2011. Indoor tracking for mission critical scenarios: A survey. Pervasive and Mobile Computing 7(1): 115.

Girard, G., Ct, S., Zlatanova, S., Barette, Y., St-Pierre, J., \& Van Oosterom, P., 2011. Indoor pedestrian navigation using footmounted imu and portable ultrasound range sensors. Sensors 11 (8): 76067624.

Jensen, C.S., Lu, H., \& Yang, B., 2009. Graph model based indoor tracking. In Mobile Data Management: Systems, Services and Middleware, 2009 (MDM09). Tenth International Conference on: 122131.

Khider, M., Kaiser, S., Robertson, P., \& Angermann, M., 2012. The effect of maps-enhanced novel movement models on pedestrian navigation performance. In The 12th annual European Navigation Conference (ENC 2008).

Li, X., Claramunt, C., \& Ray, C., 2010. A grid graph-based model for the analysis of $2 \mathrm{~d}$ indoor spaces. Computers, Environment and Urban Systems 34(6): 532540.

Liu, L., \& Zlatanova, S., 2012. Towards a 3D network model for indoor navigation, In Urban and Regional Data Management, UDMS Annual 2011: 79-92. Taylor and Francis Group, Boca Raton, London.

Miller, L.E., 2006. Indoor navigation for first responders: a feasibility study. tech. report, National Institute of Standards and Technology, Wireless Communication Technologies Group.

Moravec, H., \& Elfes, A., 1985. High resolution maps from wide angle sonar. In Proceeding of the IEEE International Conference on Robotics and Automation: 116-121. St. Louis, MO.

Spassov, I., 2007. Algorithms for map-aided autonomous indoor pedestrian positioning and navigation. EPFL Thesis, Lausanne.

Thrun, S., Burgard, W., \& Fox, D., 2005. Probabilistic robotics. MIT press.

Tsetsos, V., Anagnostopoulos, C., Kikiras, P., \& Hadjiefthymiades, S., 2006. Semantically enriched navigation for indoor environments. International Journal of Web and Grid Services 2(4): 453478.

Verbree, E., Zlatanova, S., van Winden K.B.A., van der Laan, E.B., Makri, A., Taizhou, L., \& Haojun, A., 2013. To localise or to be localised with WiFi in the Hubei museum?, In Int. Arch. Photogramm. Re-mote Sens. Spatial Inf. Sci., XL-4/W4: 31-35.

Wikipedia, 2014a. Preferred walking speed.

http://en.wikipedia.org/wiki/Preferred_walking_ speed (29 August 2014).

Wikipedia, 2014b. Taxicab geometry.

http://en.wikipedia.org/wiki/Manhattan_distance (26 August 2014).

Worboys, M., 2011. Modeling indoor space. In Proceedings of the 3rd ACM SIGSPATIAL International Workshop on Indoor Spatial Awareness: 1-6. ACM. 